\title{
Madre-figlia: un binomio complesso
}

\section{Mother-daughter: a complex binomial}

\author{
Hanna Serkowska \\ Uniwersytet Warszawski \\ hanna.serkowska@uw.edu.pl
}

\begin{abstract}
The article examines some decisive transformations that took place in the representation of the motherdaughter relationship, reflected in texts written by Italian women writers between the early 1900s and the early 2000s. Although updated, both motherhood and mother-daughter relationship appear closely linked and anchored to problems that imprison women in various predicaments. The corpus here under analysis focuses on unusual literary genealogies.
\end{abstract}

Keywords: mother, daughter, relationship, genealogy, sisterhood

I testi analizzati conducono a riflettere su quanto l'identità della donna e il rapporto madre-figlia siano strettamente correlati alla costruzione e alla valutazione del concetto di materno. L'analisi approfondita offre, inoltre, la scoperta di simmetrie tra le varie scrittrici, che invitano a riconsiderare la tradizione letteraria, premesso che:

[...] la strategia genealogica è in un certo senso l'inverso della fissazione di una tradizione. La tradizione comporta un movimento discendente, dalle premesse alle conseguenze, dai prodromi agli epigoni, impegna chi è venuto prima a tramandare un contenuto, quando non un canone ${ }^{1}$ o peggio un'ortodossia, a chi viene dopo, e chi viene dopo

\footnotetext{
${ }^{1}$ Le studiose contestano i criteri utilizzati per la formazione del canone maschile, ma notano anche la propensione delle scrittrici a rimanere fuori e segnalare una sostanziale "estraneità alla gerarchia di valore del canone, mescolando i generi, smontandoli dall'interno, collocandosi volutamente ai margini" (Crispino, 2003, 9).
} 
a recepirlo, mantenerlo e tramandarlo a sua volta, facendosene tramite obbediente [...]. La genealogia, viceversa, comporta un movimento ascendente, in cui chi viene dopo, interroga chi è venuto prima, [...] lo/la reinterpreta sulla base delle proprie domande [...]; e in questo caso è il presente che riscrive il passato (Dominijanni, 2002, 191).

Sebbene le donne, storicamente, siano state spesso rappresentate quasi esclusivamente come genitrici, è lungi dall'essere univoca la funzione reale della madre (Saraceno, 2017, 21). Giorgio osserva che nella letteratura e nella società italiana del 900 il ruolo materno è stato un archetipo potente, fatto di autosacrificio, possesso, sofferenza e resistenza, da parte di chi fungeva da pilastro dell'intera famiglia, ma che, in cambio della dedizione, esigeva dai figli lealtà e affetto esclusivi per tutta la vita (9).

Wood (232) descrive il rapporto madre-figlia, nelle opere di scrittrici, come stretto tra il mito di Clitennestra e Elettra, e ne spiega il rapporto avvelenato, citando De Beauvoir che, nel Secondo sesso, chiama in causa l'aspettativa proiettata dalla madre sulla figlia, non come un suo Altro (applicata al figlio maschio), ma come un doppio identico, per cui, quando la figlia diventa donna e si distingue dalla madre, la madre si sente tradita. Il doppio legame rappresenta il punto critico di questa relazione: la figlia deve differenziarsi dalla madre e al contempo identificarsi con il suo corpo.

Nel contesto letterario più recente il materno diviene sempre meno un argomento marginale, così come presagito da Cavarero ("la relazione madre-figlia non si ritrova nella cultura”, 2009, 47) e Muraro: „Prima del movimento politico delle donne $[. .$.$] [i]l mio rapporto con la madre era muto. [...] la nostra civiltà non è fatta$ perché una donna impari questo amore" (12). Brogi, oggi sottolinea come "il rapporto madre-figlia tende a rappresentare, con un'intensità mai smorzata, il nucleo generativo della storia narrata non solo in Italia, ma universalmente" (18).

La maternità, intesa come problema centrale delle donne, è affrontata nel 1906 da Aleramo, che va contro la moralità e l'istituzione della famiglia, nel rifiutare il ruolo di madre e di moglie legato alla "resa, apatia, debolezza, dolore e follia"2. La scelta che "una donna" compie di andare via di casa, separandosi dal marito, quando la legge non lo prevedeva (le viene tolto il figlio) e senza l'autorizzazione del marito, incapace di riscuotere l'eredità di suo zio, resta proprietà dell'uomo: „dovevo stimarmi fortunata che egli non mi facesse ricondurre colla forza” (217), si dimostra l'unica in una società fondata sui valori patriarcali che nella maternità esalta il sacrificio. Padre fedifrago, madre semifolle perché scrittrice mancata:

\footnotetext{
${ }^{2}$ L'idea della maternità come un'esperienza richiedente l'annullamento di sé, era quindi ben consolidata prima dell'avvento del fascismo la cui ideologia disegnava la „nuova mistica della donna patriottica" (Rasy, [1984] 2000, 87). Il regime non riusciva a condizionare le nascite, calate in quel periodo (le storiche parlano perciò di sciopero demografico) e pur glorificando le madri, le riconsegnava al vecchio ruolo di custodi dei figli, spose del soldato.
} 
modello di famiglia in cui "una donna" rifiuta di riprodurre, perché l'immolazione materna è una „mostruosa catena” che va spezzata, per dare ai propri figli un esempio di dignità (51). All'epoca in cui occorreva „riformare la coscienza dell'uomo, creare quella della donna" (156) il tentativo di andare oltre l'inviso aut-aut era mera velleità. Il risveglio della coscienza e la ribellione della donna avviene solo tramite la cultura (,trovai in un libro una causa di salvezza”, 99): "una donna" diventa una scrittrice, impara a camminar da sola, e così si scopre capace di conciliare il suo essere madre con la scrittura, che valorizza la vita. La cultura della donna è importante perché porta a soluzioni di equilibrio sociale, ma soprattutto perché chi educa i figli non deve essere ignorante.

Le conseguenze della gravidanza indesiderata, non solo per la donna ostracizzata nel suo ambiente, ma per il figlio che essa sta per dare alla luce, sono contemplate da Ginzburg, nelle cui opere la donna-madre vive afflitta dai sensi di colpa (La strada che va in città, 1942), si suicida (La madre, 1948), impazzisce dopo la morte della

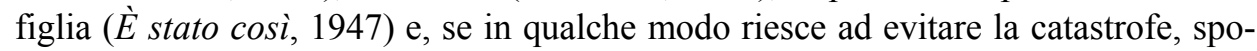
sando un altro uomo che si occupa del figlio di lei e creando un nucleo familiare anomalo, "vive una vita di un insetto attaccato alla foglia" (Tutti i nostri ieri, 1952).

C'è tuttavia chi precede Ginzburg e ritrae una serie di contraddizioni legate alla maternità nel contesto sociale, rimettendo in discussione l'identità della donna. Le giovani protagoniste di de Céspedes (Nessuno torna indietro, 1936), obbligate dalle famiglie a vivere e studiare in un istituto femminile gestito dalle suore, di notte si riuniscono per fumare, discutere degli studi, dei loro professori e di appuntamenti galanti. Lasciato il collegio, ognuna sceglie la propria strada, non "torna indietro". È impossibile dimenticare di essere state padrone di se stesse per poi rinunciarvi, ma l'emancipazione comporta un prezzo: Emanuela, che rappresenta la maternità ostacolata, rimane incinta e viene allontanata da casa e nascosta, per celare la vergogna che seppellirebbe l'intera famiglia; ma poi, nel finale, aperto e utopico, dopo la guarigione della piccola Stefania, trova la forza di riconoscere la propria identità di madre. Abbandonata dal fidanzato, dopo avergli rivelato di avere una figlia, sale con la piccola a bordo di una nave, per fare il giro del mondo.

In Dalla parte di lei (1939) si racconta invece, non della donna che sta per diventare madre, bensì del vissuto ripercorso a ritroso dalla figlia: la sua infanzia, il rapporto con la madre Eleonora, gli studi, l'amore, il matrimonio e la vita coniugale, le morti, suicidi e omicidi. Alessandra conserva il ricordo di un'infanzia solitaria, addolcita dalle letture che la madre, insegnante di pianoforte, le sceglieva (,oggi posso giudicare che la scelta di tali libri era ottima e suggerita da una solida cultura", 309).

A de Céspedes si deve il modello - seguito da Sapienza e Matteucci - di madre anaffettiva e/o assente; professionista di grande cultura, che trasmette alla figlia. Morta suicida perché innamoratasi di un giovane, passa alla figlia Alessandra anche il suo sogno d'amore ("potrei rimproverarle di avermi fatto vivere continuamente in 
un clima di esaltazione che mi aveva resa devota, innanzi tutto, al mito del grande amore, e così avermi ridotto, pur senza volerlo, alla dolorosa condizione di oggi”, 394), come fuga dallo squallore quotidiano della vita matrimoniale ${ }^{3}$. Soppresso l'istinto di fuga con l'amato, consapevole che il marito non le avrebbe permesso di portare la figlia con sé, Eleonora si getta nel fiume e annega. Una volta adulta e, a sua volta sposata, Alessandra, non potendo più sopportare l'indifferenza del marito Francesco, lo uccide. Una notte, in carcere, rivede in sogno il marito che, diversamente da come era in realtà, appare non più quell'uomo assorbito dall'attività politica, sempre girato di spalle come un muro di pietra, frettoloso e distratto, che tanto la faceva soffrire ma, al contrario, come un compagno affettuoso, mai stanco di guardarla e di discorrere con lei, come succedeva nei primi tempi della loro relazione. In questo caso, la figlia paga il prezzo della propria trasgressione, ma vive anche un tentativo di correggere il vissuto e l'auto sacrificio materni.

Oltre che Sapienza ne L'arte della gioia (v. infra), si trovano significativi collegamenti anche in Matteucci, con Costellazione familiare, la storia tragico-grottesca di una famiglia, le cui due principali figure sono la madre e la figlia. Raffaella, la madre, che è stata educata al rigore, alla disciplina e al buonsenso da una Fräulein bavarese, ha tre passioni: leggere, dar da mangiare al cane e negare il cibo alla figlia. Rosa, la figlia, che è anche la voce narrante, viene educata come un cane, con la formula: „Fuss!, sì, no, brava, e così ha imparato ad amare ciecamente la madrepadrona senza mai giudicarla" (30) e deve rassegnarsi a una madre tutt'altro che affettuosa: „Non mi ha mai abbracciata, baciata, né mi ha mai accarezzato il viso, lavato le manine, infilato calzette e scarpine. Non mi ha mai allattato né imboccata" (28).

Ma quando sopraggiungono la vecchiaia, la malattia e poi la morte di Raffaella, nella memoria della figlia si anima un volto differente della madre, quella madre che, seppur parca di affetto e carezze, l'ha comunque nutrita con un cibo immateriale, trasmettendole l'amore per la cultura. Nata ,in un'epoca in cui le ambizioni femminili si realizzavano solo nel matrimonio" e „lo studio era considerato un inutile quando non pericoloso strumento d'affrancamento del rigido canone della subordinazione donnesca" (30), Raffaella ha letto e ha fatto leggere la figlia. La maternità, passata attraverso la cultura, come scrive Aleramo, diventa una fonte di forza, e la figlia un essere umano "intero".

Come Maternità, di Negri, anche Lettera a un bambino mai nato di Fallaci, assume la forma di dialogo tra la gestante e il figlio che porta in grembo. Dialogo apparente, in verità un'introspezione, in quanto l'embrione-interlocutore non varca le prime fasi di sviluppo, e non interagisce con la madre, se non in via immaginaria. Fallaci sottolinea in questo modo che alla donna non appartiene nulla, neanche il suo

\footnotetext{
${ }^{3}$ Questo vero e proprio bovarismo riuscirà a infrangere solo Modesta de L'arte della gioia di Sapienza, scindendo il matrimonio dall'amore romantico.
} 
stesso corpo, eppure le spetta l'orribile facoltà di decidere, di scegliere da sola per due, madre e figlio, mentre l'altro è muto. Fallaci passa al vaglio i sentimenti contraddittori di chi sta per diventare madre, di colei che si pone il problema di dare vita o di negarla, non tanto per rivendicare la propria libertà di scelta, quanto per capovolgere il discorso e togliere l'onnipotenza, insita in tale apostrofe. La gravidanza fallita, l'aborto spontaneo, tolgono il potere di generare e riducono le scelte al negativo: la donna può interrompere la gravidanza in modo attivo, se questa è la sua decisione, ma non tenerla. L'incisività del libro di Fallaci è rafforzata dal momento storico in cui è stato scritto: sono gli anni che precedono la legalizzazione dell'aborto („,bambino, metterti al mondo, giuro, non mi diverte”, 7), anni dell'ideologizzazione della maternità, in cui si è discusso animatamente sulle conseguenze del diventare madre per una donna professionista e sui minori diritti delle donne rispetto agli uomini („Cos'è questo tuo diritto di esistere che non tiene conto del mio diritto di esistere?", 56). La gravidanza si rivela problematica, quanto superflua per la donna („Te l'ho già detto che non ho bisogno di te”, 8), la cui identità non dipende dal suo diventare madre o meno. In un immaginario processo a cui si vede sottoposta in veste di imputata, la gestante sente le accuse del medico, dei genitori, del capo, e delle amiche femministe, che si basano, a seconda di chi, di volta in volta le proferisce, ora sul diritto del bambino di nascere, ora sul diritto della donna a una vita autonoma. La conclusione è volutamente ambigua. Perciò (Di Rollo, 2016) Fallaci immagina la sua protagonista interloquire con un figlio maschio - portavoce della società patriarcale che la condanna. Dati quei vincoli e limiti, fatti propri, la donna che non diventa madre non ha il diritto di (continuare a) vivere, di qui la morte finale della protagonista.

Nel luglio del 1970 la Rivolta Femminile firmava il Manifesto contenente pregnanti clausole in cui si individuava il primo motivo di rancore della donna verso la società ,nell'essere costretta ad affrontare la maternità come aut-aut” (Lonzi, 1974, 13). Su tale sfondo risalta la particolarità dei romanzi di Lagorio, Un ciclone chiamato Titti e La spiaggia del lupo, che vanno contro corrente, rispetto alle coeve rivendicazioni femministe e all'idea di incompatibilità della maternità con la neoacquisita autocoscienza e con il diritto all'aborto. La protagonista de La spiaggia del lupo decide di avere il figlio nato da un rapporto con un quasi estraneo, sostenendo tutte le difficoltà che la scelta di diventare ragazza-madre comporta. Il racconto non si ferma sulla soglia della nuova vita, come Nessuno torna indietro di de Céspedes. Seppur giovane e inesperta, Angela trova la forza di affrontare la gravidanza da sola, con gli stenti che ciò implica. In sintonia con lo spirito dei tempi paiono invece le opere scritte da Marina Jarre, Giuliana Ferri, Carla Cerati (1975), Lidia Ravera e Dacia Maraini. Il risveglio della protagonista di Maraini, lento e doloroso, passa attraverso la scoperta che il desiderio di avere un figlio che nutre suo marito è dettato dal bisogno di riaffermare la supremazia maschile. „Giacinto mi spia. [...] Con il figlio lui pensa che tornerò la donna dolce, remissiva, disponibile, arresa di 
prima". Il marito le dà un pugno sul petto urlando che il figlio è suo e nessuno glielo può portare via, ma Vanna non ha ,intenzione di fare un figlio non voluto, strappato a tradimento" (261-262). Il racconto si ferma poco dopo la descrizione dell'aborto che Giovanna subisce senza anestesia: „Ora sono sola e ho tutto da ricominciare” (269). Anche la protagonista di Cerati, consapevole che la maternità non è tutto, la ripudia. E il lungo monologo - nel racconto di Ravera - di una donna afflitta dal desiderio di avere un figlio, in dissidio con le esigenze di efficienza nella vita professionale e affettiva, di spregiudicatezza e seduzione femminili, mostra la maternità, non più come un obbligo, ma sempre come una trappola e un ostacolo alla carriera.

Chi non discrimina tra il suo essere donna e madre, ma anzi li percepisce come inseparabili, è il personaggio preferito di Morante. Sono figli maschi (non come in Deledda) solo perché Morante considerava il personaggio maschile più accreditato. $\mathrm{Ne}$ L'Isola di Arturo è vistosa la predilezione della scrittrice, in programmatico disaccordo con i coevi femminismi, per la madre oblativa, un'analfabeta del Sud che va in giro scalza, vestita di nero e che si annulla nella la propria creatura, una madre mitizzata, divenuta animale ne La Storia, vegetale in Aracoeli (il giardino di Almendral, mandorleto-paradiso, l'Eden materno). Chi (Ida de La Storia) però cerca di sfamare i piccoli come una tigre che si strappa coi denti dal proprio corpo brandelli di carne per darli da mangiare al cucciolo e quando questi muore, impazzisce, e chi (Aracoeli), dopo la morte della secondogenita, va alla deriva e, dopo aver costretto suo figlio Manuele ad assistere alle sue intemperanze, esce di scena. Lentamente viene meno la diade madre-figlio, matrioska che risucchia il mondo. È preclusa l'androginia anelata dal niñomadrero come gemellaggio con la madre. La madre, intensamente amata, desiderata, infine demonizzata, annuncia al figlio che la idoleggia la fine del suo amore: „Sei sempre il mio niño? Sei sempre il niño mio? [...] E invece, io non so più la tua mamita" (1377) e lo costringe a dubitare che quell'amore smisurato ci fosse, o se era tutto uno scherzo.

„Mia madre e io abbiamo vissuto a lungo sole, innamorate senza rimedio l'una dell'altra". Inizia così Passaggio in ombra di Di Lascia (14) che emula Morante nell'ambivalente adorazione materna. Come in molti altri racconti sul rapporto madre-figlia, la figlia narra fatti già consumati, azioni compiute dopo la scomparsa della madre. Quarantenne, Chiara ricorda le dolci impronte di baci che Anita le stampava sulla bocca e sui capelli, lo stare in braccio alla madre come in un nido amoroso, da cui guardare il mondo, il caldo rifugio tra il seno e l'ascella materna, dove scivolare e cullarsi nel sonno. Ma, come per il Manuele morantiano, il ricordo dell'amore disinteressato e spontaneo è l'unico riparo dalla condanna presente, l'isola d'amore, l'unico limbo felice. Quando la madre muore, la figlia, un tempo bella intelligente e laboriosa, smette di agire. Passa nell'ombra.

Ancora, nelle opere degli anni 80 e 90, la figlia muove dalla morte/scomparsa della genitrice, tentando di riportarla in vita tramite la scrittura (Sanvitale), o di 
riconciliarsi con essa, riconoscendone perfino la sessualità (Ramondino, Ferrante ne L'amore molesto). La figlia Sonia (in Sanvitale), frutto di una relazione illegittima che la madre Marianna intrattiene con un ufficiale dell'esercito, sposato con un'altra, e che le costringe a una vita di indigenza e nomadismo, si scopre con-fusa con la madre al punto da non sapersene più distinguere (il bambino muore dentro il grembo di Sonia nello stesso momento in cui la madre muore di cancro), con la conseguente sensazione di odio, invidia e desiderio del corpo materno. La madre, in Ramondino, mette in moto un sistema di inibizioni, ma non riesce a impedire alla figlia di tramutarsi in un suo doppio („ognuno di noi ha un altro se stesso”, 261). S'innesca uno scambio di ruoli, in conseguenza al quale la figlia si sente "quasi una madre per lei" (160), mentre la madre si rivela „una madre bambina, compagna di giochi” (39). Alla figlia che capisce l'influsso della madre, già assente nella propria vita, spetta il compito di costruire in modo diverso il proprio rapporto con la maternità, attraverso ricordi, memorie, sogni.

Il romanzo di Cerati (1996) si dipana a partire dalla morte della madre, come quello di Jarre (1987) e di Sereni. La riconciliazione con la madre si compie, rivisitandone a ritroso il vissuto e riconfigurandone il proprio. Quando le tocca di occuparsi della madre anziana e rimasta sola, Giulia si sente dibattuta tra l'obbligo di cura e l'avversione che riuscirà a superare solo spingendo la madre a raccontarle la sua vita. La madre si muta in una sofferente protagonista, mentre nella figlia, trasformata in amorevole testimone, si sprigiona un immenso amore. Capisce di averlo sempre avuto per la genitrice: ,soltanto oggi mi rendo conto di quanto posto abbia occupato questa donna, mia madre, malgrado io non glielo volessi concedere, e di come la sua figura si sia ingigantita nei ricordi" (Cerati, 1996, 263).

Il romanzo di esordio di Ferrante (1992) si apre con il ritorno dal Nord per i funerali di Amalia, forse suicida, della figlia, la quale percepisce la propria incapacità di procreare come conseguenza del rapporto irrisolto con sua madre: aveva sempre rifiutato il corpo piacente e allegro della madre perché attraeva gli sguardi maschili. Ora Delia non la rifugge più, si identifica con essa indossandone gli abiti, guardandone la foto sulla carta d'identità dice: „mi guardai, mi sorrisi. [...] Amalia c'era stata. Io ero Amalia" (178). Anziché di con-fusione, de Rogatis parla qui della sostituzione della madre, reinventata a seguito di un rito trasformativo (52), ma il minaccioso corpo-a-corpo con la madre, inteso come con-fusione, luogo di follia, a angoscia e regressione (Buzzatti, Salvo, 1995) risalta ancora di più nel secondo romanzo (2002). La con-fusione madre-figlia comporta il rischio evocato dalla figura dell'atavica "poverella" - madre e donna abbandonata che finisce folle o suicida: "oggi tocca a me, tra un attimo a mia figlia, era successo a mia madre, a tutte le mie antenate $[\ldots]$ succederà" (98). Solo nel terzo romanzo di Ferrante (2006) si avverte un desiderio spasmodico di vivere la maternità al positivo, libera dalle tare del passato. L'assistere a quel che in un primo momento si raffigura come un idillio tra madre e figlia, culmina in una pulsione di violenza provocata dal ricordo della 
propria maternità, il cui bilancio è tutt'altro che positivo. Leda ruba l'adorata bambola con cui giocano Nina e Elena e poi, in una scena che sfiora l'orrore, "sgrava" il pupazzo. Il ricordo delle proprie mancanze di madre, che aveva abbandonato le figlie per quattro anni e del difficile rapporto con la progenie da allora in poi, manifesto nelle rare telefonate e conversazioni bloccate, fa trapelare colpa e risentimento, ma in seguito sana le antiche piaghe. Il romanzo si chiude sul limine di un nuovo, sereno rapporto con le figlie. Le immagini della maternità minacciosamente riemersa ${ }^{4}$, soggetta alla trasformazione come in una fiaba, fanno sortire

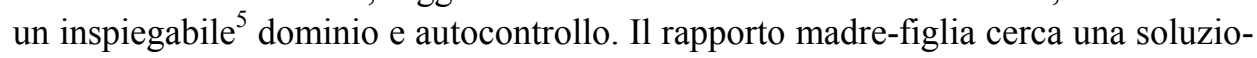
ne anche nella tetralogia de $L$ 'amica geniale. Vi si rappresenta una riconciliazione definitiva: il corpo materno, prima malato e poco dopo morto, viene sussunto dentro il proprio. Al termine di un'ostilità e di un disprezzo verso la madre, durati tutta la vita e determinati anche dalla paura della confusione: „starle vicino significava restare nel suo mondo, diventare nel tutto simile a lei” (I, 318); ,avevo temuto che il passo di mia madre mi avesse raggiunto" (III, 213-214), Elena inizia a provare tenerezza, mentre il corpo materno trasloca per prendere dimora dentro il suo. Sente un acuto dolore nel fianco, ma non teme più di diventare zoppa come la madre. Scopre un modo diverso di amarla, di stare con lei dopo che questa viene a mancare. Decide di non curarsi e di coltivarsi ,quel fastidio, come un lascito custodito nel mio stesso corpo" (IV, 207). Il viaggio nel tempo si compie a ritroso: segue una temporalità reversibile, ciclica, genealogica per l'appunto: ma non è la madre a cercare la figlia (come fa Demetra a Kore), bensì il contrario.

La tipologia di Recalcati (2015) - madri-Madonna e madri-Medea, madri-coccodrille, che nel sacrificarsi imprigionano il figlio in un'eterna gravidanza, e madrinarcise, troppo occupate a perseguire il successo nel lavoro e a coltivare relazioni personali per non trascurare il figlio, non tiene conto delle ultime puntate sul materno, scritte dalle giovani donne senza lavoro, senza famiglia, madri single, madri con figli adottivi, o madri divenute tali grazie alla fecondazione assistita. I drammi dell'infertilità e la tragica consapevolezza che desiderare un figlio non è lecito fanno scattare nei confronti della madre sentimenti di invidia e di impotenza. Basti citare la nuova letteratura del lavoro, affollata di racconti sul mondo delle precarie, scritti spesso da esordienti sul filo dell'autobiografia, esperienze vissute e reportage di denuncia che raccontano una generazione di trentenni prive di futuro, figlie e nipoti delle sessantottine che non andavano d'accordo con la propria madre

\footnotetext{
${ }^{4}$ Le immagini di procreazione-gravidanza-maternità assediano e esasperano la sofferenza di Leda: madre e figlia, Nina e Elena giocano a "mettere incinta" la bambola immettendole nella pancia un insetto, Rosaria incinta ostenta il suo ventre in modo tra invadente e trionfante.

${ }^{5}$ De Rogatis spiega l'enigma con un impulso di uccidere simbolicamente la madre represso, bloccato. La figlia riesce a riparare la madre dalla propria aggressione, ribellione e protesta contro l'eredità (88).
} 
e buttavano via quel che oggi è un traguardo agognato e quasi insperabile: il lavoro fisso e retribuito, le vacanze, la famiglia, la maternità.

Le donne fanno più fatica. In una condizione di precariato te lo scordi di mettere al mondo un figlio, questa è la verità. E chi i figli li ha fatti, si trova magari a rendere conto ai propri bambini delle proprie nevrosi, delle proprie insicurezze.

Oggi fare un figlio è un lusso. Un lusso inaudito. [...] Sentire che il bisogno di avere un bambino, oggi, è semplicemente illegittimo. Perché tutti te lo ripetono. Perché tu stessa hai interiorizzato questa cosa (Nove, 2006, 9 e 11).

De L'arte della gioia di Sapienza, scritto nell'arco di nove anni (1967-1976), ma pubblicato solo nel $1994^{6}$, si sottolinea la precocità delle idee, legate soprattutto ai rapporti di genere, alla costruzione del materno e all'esplorazione dell'identità femminile, sullo sfondo dei rapporti sociali coevi: "gli spazi patriarcali messi a nudo" (Wehling-Giorgi, 2017), che vincolano quei ruoli e quelle identità. Sapienza sarebbe in anticipo sul suo tempo nel raffigurare la figlia che guarda la madre, con un misto di amore e risentimento, consapevole della complessa eredità, determinata dal contesto patriarcale che complica o riduce al silenzio la relazione madre-figlia. Il romanzo esplora il rapporto conflittuale con il materno - funzione, intesa tradizionalmente, non da seguire, ma da rigettare in tronco. Modesta ricopre un ruolo attivo nella morte delle sue tre madri. La figlia impara da ciascuna madre quanto quella è in grado di trasmetterle e toglie loro tutto, perfino la vita. La madre biologica, tutta cultura e politica (era l'unica donna all'epoca che studiava, e allora doveva studiare anche sua figlia) e niente calore, affetto o nutrimento, indirettamente responsabile dello stupro che Modesta subisce all'età di appena di nove anni, violentata da chi le viene presentato come suo padre, muore nell'incendio provocato dalla figlia in fuga. Leonora, la madre superiora nel convento dove la piccola Modesta si ripara, orfana, disonorata e senzatetto, e che ne sarà la madre culturale e spirituale (farà studiare la ragazza di cui riconosce l'intelligenza), viene uccisa perché Modesta non vuole farsi monaca. Viene assassinata anche la madre sociale di Modesta, la vecchia matriarca Gaia (di fatto la madre di Leonora), donna forte e volitiva come un uomo, eliminata per potersi impadronire della sua posizione, del suo potere e dei suoi beni. A fronte di questa tormentata storia, la finale riconciliazione con la propria maternità stupisce, tanto per la mancanza di modello di maternità da seguire, quanto per la sua stessa inedita costruzione. La nascita di una diversa, non normata costellazione familiare e di un nuovo rapporto madre-figlia, non sarebbe possibile senza sfidare l'ordine simbolico dominato dal maschio e senza decostruire i ruoli di genere.

\footnotetext{
${ }^{6}$ Gli ultimi anni segnano una vera e propria "Goliarda fever" - l'operazione Sapienza imita quella precedente, dedicata a Ferrante (v. E. Cooke, Disobedience is a Virtue in The New Yorker, 23 gennaio 2014) - con una marea di saggi sparsi e perfino in volume monografico Goliarda Sapienza in Context (2016).
} 
Modesta vive una vita sregolata e costituisce un nucleo familiare, caratterizzato da non convenzionali legami di parentela, ma ne sfrutta lo statuto protettivo e legittimante. Scarparo e Di Rollo (2015) affermano che vi avvenga il passaggio da un sistema di rapporti gerarchici tra donne, modellati sul sistema di potere maschile (una impartisce all'altra il sapere e il potere, una plasma l'altra a suo piacimento), a un altro di autorità e autorevolezza, paritario, non oppressivo, ma non occorre dimenticare che quest'ultimo, se porta ad assoluta libertà, o anarchia, pace e armonia, poggia su un fulcro di violenza, prevaricazione, crimine, tanto più ripugnanti, in quanto rivolti contro le proprie benefattrici. Disarcionando questa incongruenza, occorre riconoscere che Modesta riesce a diventare una figura autorevole per le proprie figlie, assumendo il ruolo di madre e di padre, rendendole indipendenti anche economicamente, preservandole da norme e convenzioni costituite. Ciò ne fa una madre, nella letteratura italiana, bizzarra, eccentrica (Fortini, 2016).

Parrella ne Lo spazio bianco aggiorna il monologo-lettera di Fallaci (Di Rollo, 2016). La donna che sta per diventare madre a seguito di una gravidanza non pianificata, ma forte e indipendente, affronta la maternità da sola e considera l'opportunità di mettere al mondo una creatura come scelta. Il contesto socio-culturale ormai cambiato fa sì che con la morte del feto nel testo di Fallaci, anche la donna perda tutto e perfino ne muoia, mentre la nascita prematura della figlia, nel testo di Parrella, non mette a rischio la vita della madre, non ne sbarra la futura carriera, ma è opportunità di felicità e gioia. L'essere donna per la protagonista di Parrella non dipende dal suo diventare madre. Oltre alla maternità, essa ha altri elementi per definirsi donna. Il suo essere madre dipende solo dal fatto se la nata prematura sopravvive o meno.

Con la fecondazione assistita e con le possibilità pressoché illimitate di adozione, la maternità separata dalla sessualità fa nutrire fantasie di onnipotenza (Fraire, 2017, 35), ma richiede definizioni. Il bambino può essere generato da tre madri, che se ne ripartiscono le funzioni: la madre genetica, donatrice di ovuli, la madre portatrice, che offre in affitto il proprio utero e la madre sociale, che adotta e cresce il bambino. Alla luce dei recenti sviluppi nel campo delle biotecnologie e della Procreazione Medicalmente Assistita, non si può più pensare che solo i legami di sangue garantiscano una relazione serena e sicura, mentre al di fuori di essi si insidino per il figlio pericoli vari - quell'idea portava al "familismo perverso" e alla condanna senza scampo di chi è estraneo al nucleo familiare (Pagliano, 1993, 37). Un esempio di maternità non legata alla genealogia, all'ereditarietà o alla verticalità, ma fatta dipendere dalla filiazione elettiva, d'anima ce la offre Murgia in Accabadora. Un legame madre-figlia non di sangue, ma affettivo. Di cura reciproca e amore.

Qualche volta, il rifiuto della madre e la genealogia discontinua fanno intrecciare rapporti verticali nipote-nonna, come se gli anni della ribellione delle figlie contro le madri avesse inferto una ferita troppo profonda per essere placata. La nonna, spesso diversissima in tutto dalla madre, ne fa le veci. Ma un esempio di maternità del tutto 
non normativa la si trova nei legami orizzontali di sorellanza (Daly, 1978), legami che spostano in modo radicale i confini del materno. Fondata su parentela tra donne, o su un rapporto di intimità nel senso di solidarietà, comunità di esperienze, o su un elemento di autoaffermazione e identità della donna, sisterhood è 1'ultima risposta all'irrisolto concetto del materno che smentisce il rapporto di affidamento ideato all'interno della Diotima - verticale, gerarchico, asimmetrico tra una mentrice-guida e una discente che riconosce l'autorità e l'autorevolezza della maestra-quasi-madre. La sorellanza, per converso, è un legame paritario come tra "comadri" (Pinkola Estès), o amiche che si stimano e che fungono anche da madri affettuose l'una per l'altra. Si proteggono, si ascoltano e si guidano in diversi aspetti della vita, si prendono cura e si fidano l'una dell'altra. Qualcosa di simile si trova anche ne L'Arminuta di Di Pietranatonio. Le due ragazze, poi divenute donne, sorelle per davvero, cresciute separatamente per alcuni anni, poi rimesse insieme - Arminuta (ritornata, restituita - così viene chiamata nel paese abruzzese dove è rispedita) e la sua sorella minore Adriana, sono l'una per l'altra come una madre. Una madre di cui fidarsi, che non abbandona mai, diversamente dalle due madri adulte di cui Arminuta si sente orfana. Orfana della madre biologica, senza nome, come sua figlia, che l'aveva data via, e della madre sostitutiva, Adalgisa, che la voleva appena nata perché da sola non riusciva a concepire, ma che poi decide di rispedire senza spiegazione, con il pretesto che la famiglia di origine l'aveva richiesta, come un pacco. Il mistero che tormenta per alcuni anni Arminuta, il timore che Adalgisa sia malata o morta, si spiega con il divorzio della seconda madre e le nuove nozze dopo essere rimasta incinta di un altro. Fallimentari nel loro ruolo, le due madri, si eclissano, ma la delusione per l'incapacità di entrambe le figure materne non impedisce che si generi in Arminuta un istinto di protezione verso la sorella minore e il fratellino handicappato, messo in un istituto, di cui entrambe continueranno a prendersi cura. La storia si chiude con la scena in cui le sorelle lasciano, mano nella mano, la casa di Adalgisa che maldestramente tenta di riallacciare il legame con la figlia adottiva, ormai decaduto. Si avviano „,così sole e vicine” sulla spiaggia, insieme si immergono nell'acqua: „Mia sorella. [...] Da lei ho appreso la resistenza. [...] Nella complicità ci siamo salvate" (136).

I testi qui esaminati usano quasi tutti l'analessi: nascono dopo la scompar$\mathrm{sa} /$ morte della madre e sono scritti in un contesto storico-sociale mutato. Il fatto che la maternità diventi materia di racconti, tradotta in sapere e vita (Muraro, 1992, 18) conferma l'esigenza di imparare ad amare la madre, ripensando il rapporto madrefiglia e il vissuto materno. Le figlie hanno percorso la strada che va dalla critica della maternità come ruolo unico a cui è d'obbligo tendere, con inviso sacrificio e poi visto come scelta ambigua, all'elogio di maternità eterogenea e eteronormativa, d'accordo con la molteplicità di fattori che determinano le pratiche della maternità, libera del sentimento di colpa o inanità. La maternità aproblematica non è concepibile, ma ci sono più modi di essere madri, costituire nuclei familiari 'ricostruiti' 
(patchwork families) e anche senza diventarlo si può essere persone intere. A guardare avanti è l'utopia della sorellanza (comadri) che elude la figura e l'ordine gerarchico della madre - nel far sì disinnesca meccanismi difensivi e di competizione insiti perfino nell'idea di affidamento, ma non il "pensiero materno". Valorizza le doti di cura e accudimento senza riprodurre il rapporto di potere. I testi letti in quest'ottica si risistemano rispetto ai canoni di tradizione stabiliti, scoprono una genealogia, una costellazione fatta di nuclei inediti, eccentrici, dissonanti, bizzarri reversibile, ciclica per cui le figlie di Morante sono Di Lascia e Di Pietrantonio, di Aleramo - Fallaci e Parrella, di de Céspedes - Matteucci, mentre Sapienza si ricollega a molte madri tra cui forse de Céspedes, Banti, Aleramo e chi l'ha preceduta. 


\section{BIBLIOGRAFIA}

Aleramo, S. ([1906] 1995). Una donna. Milano: Feltrinelli.

Bazzoni, A., Bond, E., Wehling-Giorgi, K. (Eds.). (2016). Goliarda Sapienza in Context: Intertextual Relationships with Italian and European Culture. Vancouver BC: Fairleigh Dickinson University Press.

Benedetti, L. (2007). The Tigress in the Snow. Motherhood and Literature in Twentieth-Century Italy, Toronto: Toronto University Press.

Brogi, D. (2017). Per un nuovo racconto di formazione. In D. Brogi et al. (Eds.), Nel nome della madre (pp. 9-19). Roma: Del Vecchio Editore.

Brogi, D., de Rogatis, T., Franco, C., Spera, L. (2017). Nel nome della madre. Roma: Del Vecchio.

Buzzatti, G., Salvo, A. (Eds.). (1995). Corpo a corpo. Madre e figlia nella psicanalisi. Roma-Bari: Laterza.

Cavarero, A. ([1990] 2009). Nonostante Platone. Figure femminili nella filosofia antica. Verona: Ombre Corte.

Cavarero, A. (2007). Il femminile negato. Rimini: Pazzini.

Cerati, C. (1975). Un matrimonio perfetto. Milano: Frassinelli.

Cerati, C. (1996). La cattiva figlia. Milano: Frassinelli.

de Céspedes, A. (2011). Romanzi, a cura di M. Zancan, con la collab. di S. Ciminari, L. Di Nicola e M.C. Storini. Milano: Mondadori (Nessuno torna indietro, 1936; Dalla parte di lei, 1949).

Crispino, A.M. (Ed.). (2003). Oltrecanone. Per una cartografia della scrittura femminile. Roma: Manifesto.

Daly, M. (1978). Gyn/Ecology. The Metaethics of Radical Feminist. Boston: Beacon Press.

Di Lascia, M. (1995). Passaggio in ombra. Milano: Feltrinelli. Céspedes

Di Pietrantonio, D. (2017). L'Arminuta. Torino: Einaudi.

Di Rollo, A. (2016). Motherhood and Female Identity in Oriana Fallaci and Valeria Parrella: A Case of Literary Maternage? Intervalla: special vol. 1, 35-46.

Dominijanni, I. (2002). Nella piega del presente. In Diotima (Ed.), Approfittare dell'assenza. Punti di avvistamento sulla tradizione (pp. 187-212). Napoli: Liguori.

Fallaci, O. ([1975] 2009). Lettera a un bambino mai nato. Milano: Rizzoli.

Ferrante, E. (1992). L'amore molesto. Roma: e/o.

Ferrante, E. (2002). I giorni dell'abbandono. Roma: e/o.

Ferrante, E. (2006). La figlia oscura. Roma: e/o.

Ferrante, E. (2011). L'amica geniale. Roma: e/o [I].

Ferrante, E. (2012). Storia del nuovo cognome. Roma: e/o [II].

Ferrante, E. (2013). Storia della bambina perduta. Roma: e/o [IV].

Ferrante, E. (2013). Storia di chi fugge e di chi resta. Roma: e/o [III].

Ferri, G. (1973). Un quarto di donna. Venezia: Marsilio.

Fortini, L. (2016). Beyond the Canon. Goliarda Sapienza and Twentieth-Century Italian Literary Tradition. In A. Bazzoni, E. Bond, K. Wehling-Giorgi (Eds.), Goliarda Sapienza in Context: Intertextual Relationships with Italian and European Culture (pp. 131-145). Vancouver BC: Fairleigh Dickinson University Press

Fraire, M. (2017). All'origine la procreatrice. In D. Brogi, T. de Rogatis, C. Franco, L. Spera (Eds.), Nel nome della madre (pp. 31-42). Roma: Del Vecchio.

Ginzburg, N. (2001). Opere (vol. 2), a cura di C. Garboli. Milano: Mondadori (La strada che va in città, 1942; È stato così, 1947; La madre, 1948; Tutti i nostri ieri, 1952).

Giorgio, A. (2002). Mothers and daughters in Europe: mapping the territory. In A. Giorgio (Ed.), Writing Mothers and Daughters. Renegotiating the Mother in Western European Narratives by Women (pp. 1-9 ). New York: Berghahn. 
Giorgio, A. (2002). Rappresentare la madre: temi e problemi in un secolo di narrati. In I. Fried, E. Baratono (Eds.), Il Novecento. Un secolo di cultura: Italia e Ungheria (pp. 131-152). Budapest: Eötvös Loránd University TFK.

Jarre, M. (1971). Negli occhi di una ragazza. Torino: Einaudi.

Lagorio, G. (1969). Un ciclone chiamato Titti. Rocca San Casciano: Cappelli.

Lagorio, G. (1977). La spiaggia del lupo. Milano: Mondadori.

Lazzari, L., Charnley, J. (Eds.). (2016). To Be or Not to Be a Mother: Choice, Refusal, Reluctance and Conflict. Motherhood and Female Identity in Italian Literature and Culture. Intervalla: special vol. 1.

Lonzi, C. (1974). Sputiamo su Hegel. La donna clitoridea e la donna vaginale e altri scritti. Milano: Rivolta Femminile.

Maraini, D. (1975). Donna in guerra. Milano: Rizzoli.

Matteucci, R. (2016). Costellazione familiare. Milano: Adelphi.

Morante, E. (1994). Aracoeli. In C. Cecchi, C. Garboli (Eds.), Opere (vol. II). Milano: Mondadori.

Muraro, L. (1992). L'orientamento della riconoscenza. In Diotima (Ed.), Il cielo stellato dentro di noi. L'ordine simbolico della madre (pp. 9-19). Milano: La Tartaruga.

Murgia, M. (2009). Accabadora. Torino: Einuadi.

Negri, A. (1904). Maternità. Milano: Fratelli Treves.

Nove, A. (2006). Mi chiamo Roberta, ho 40 anni, guadagno 250 euro al mese. Torino: Einaudi

Pagliano, G. (1993). L'infante abbandonato e l'infante adottato. In A. Neiger (Ed.), Maternità trasgressiva e letteratura (pp. 27-37). Napoli: Liguori.

Parrella, V. (2008). Lo spazio bianco. Torino: Einuadi.

Passerini, L. ([1998] 2008). Autoritratto di gruppo. Firenze: Giunti.

Pinkola Estès, C. (2006). La danza delle grandi madri. Milano: Frassinelli.

Ramondino, F. (1981). Althénopis. Torino: Einaudi.

Rasy, E. ([1984] 2000). Le donne e la letteratura. Scrittrici, eroine e ispiratrici nel mondo delle lettere. Roma: Editori Riuniti.

Ravera, L. (1979). Bambino mio. Milano: Bompiani.

Recalcati, M. (2015). Le mani della madre. Desiderio, fantasmi ed eredità del materno. Milano: Feltrinelli.

de Rogatis, T. (2017). Ripensare l'eredità delle madri. Cerimoniale iniziatico e strutture rituali ne L'amore molesto, I giorni dell'abbandono $e$ La figlia oscura di Elena Ferrante. In D. Brogi et al., Nel nome della madre (pp. 71-91). Roma: Del Vecchio Editore.

Sanvitale, F. (1980). Madre e figlia. Torino: Einaudi.

Saraceno, Ch. (2017). Maternità: un incessante lavoro di costruzione sociale. In D. Brogi et al. (Eds.), Nel nome della madre (pp. 21-29). Roma: Del Vecchio.

Scarparo, S., Di Rollo, A. (2015). Mothers, Daughters and Family in Goliarda Sapienza's "L'arte della gioia". The Italianist, 35, 91-106.

Sereni, C. (1993). Il gioco dei regni. Firenze: Giuinti.

Spera, L. (2017). "L'anima mia è con te": figure della maternità nell'archivio personale e nella produzione narrativa di Alba de Céspedes. In D. Brogi et al. (Eds.), Nel nome della madre (pp. 129-143). Roma: Del Vecchio.

Wehling-Giorgi, K. (2017). Dislocazioni materne, linguaggio e identità femminile nelle opere di Goliarda Sapienza. In D. Brogi et al. (Eds.), Nel nome della madre (pp. 143-156). Roma: Del Vecchio.

Wood, S. (1995). Italian Women's Writing 1860-1994. London: Athlone. 